\title{
Capital asset pricing model in Portugal: Evidence from fractal regressions
}

\author{
Ladislav Kristoufek ${ }^{1}$ \& Paulo Ferreira ${ }^{2,3,4}$ (D)
}

Received: 1 November 2017 / Accepted: 6 April 2018/Published online: 18 April 2018 \# ISEG 2018

\begin{abstract}
We examine risk profiles of the Portuguese stock market index component stocks using a novel approach to the classical capital asset pricing model (CAPM). Specifically, we estimate the CAPM via fractal regressions that allow studying the marginal effects at selected scales. In this way, we can reveal whether the risk is perceived differently by market participants with different investment horizons. Apart from the analysis itself, we provide new statistical insights into the issue of separating and comparing the scale-specific effects with statistical validity. We find several stocks deviating from an expected risk perception homogeneity across investment horizons. This is true for both analysed periods, i.e. before and after the global financial crisis. There are also several stocks that changed their relationship to the market portfolio in between, which has strong implications for possible portfolio construction. The proposed methodology is not limited to financial topics but can be used in any discipline where the scale-specific marginal effects might be of interest.
\end{abstract}

Keywords Capital asset pricing model - Detrended cross-correlation analysis . Detrending moving-average cross-correlation analysis $\cdot$ Fractal regressions $\cdot$ Portugal

JEL codes $\mathrm{C} 19 \cdot \mathrm{G} 12$

\footnotetext{
* Paulo Ferreira pjsf@uevora.pt

Ladislav Kristoufek

kristouf@utia.cas.cz

1 Institute of Information Theory and Automation, Czech Academy of Sciences, Pod Vodarenskouvezi 4, CZ-18208 Prague, Czech Republic

2 VALORIZA - Research Center for Endogenous Resource Valorization, Portalegre, Portugal

3 CEFAGE-UE, IIFA, Universidade de Évora, Largo dos Colegiais 2, 7000 Évora, Portugal

4 Instituto Politécnico de Portalegre, Portalegre, Portugal
} 